\title{
Simulation Research on Detection Sensor of Metal Wear Particle in Oil Fluid
}

\author{
Haiying Jiang, Ping Gao and Wenjun Zhao \\ School of Information Science and Engineering, University of Jinan, Jinan, Shandong, China, 250022
}

\begin{abstract}
Using COMSOL Multiphysics software established finite element models of the $2 \mathrm{D}$ axis symmetric and the $3 \mathrm{D}$ for the detection sensor of metal particle. Under different models and parameters, containing particles in different positions of five models, excitation frequency, turns ratio, the distance between the coils and duct diameter etc, simulated the variation of the magnetic field of the coils, and studied the performance of the sensor. Through simulation getting the more appropriate model and other main parameters provide the important basis for sensor design.
\end{abstract}

Keywords-metal wear particle; detection sensor; COMSOL; simulation

\section{INTRODUCTION}

Mechanical equipment will produce wear in the running process, Monitoring the size and quantity of metal wear particles in lubricating oil has an important role in improving the safety of mechanical equipment. References [1] detailed analysis the principle of three models of metal wear particle detection sensors: single coil, double coils and three coils (all are air-core solenoids), and compared their respective advantages and disadvantages. A mathematical model of three coils with reverse-double-excitation coils type sensor was designed by [2]. In the references [3], the characteristics of the three coils sensor in [2] are analyzed, and the sensor is designed, which can be used to detect the wear particles larger than 125 microns in oil fluid.

This paper adopts the structure of double excitation coils type sensor [2], and applies the COMSOL Multiphysics software to establish the 2D axis symmetric and the 3D finite element models of the sensor, considering the factors such as the coil size, input voltage, turns of the coil and position of the metal particle, the magnetic field of excitation coils, the magnetic field changes during the metal particles through coils and the influence to performance of the sensor are studied. Finally, the most suitable model is obtained by simulation, and the number of turns of the coil, turn ratio, sensor structure and other important parameters are determined.

\section{THEORETICAL MODEL}

The detection sensor of metal wear particle adopts reverse double excitation coils [2] [3]. The sensor schematic is shown in Figure 1. The middle coil is the induction coil. When the metal particles flow through the sensor in the oil, the magnetic field will be disturbed to generate the induced voltage in middle coil. The sensor's induced electromotive force model is

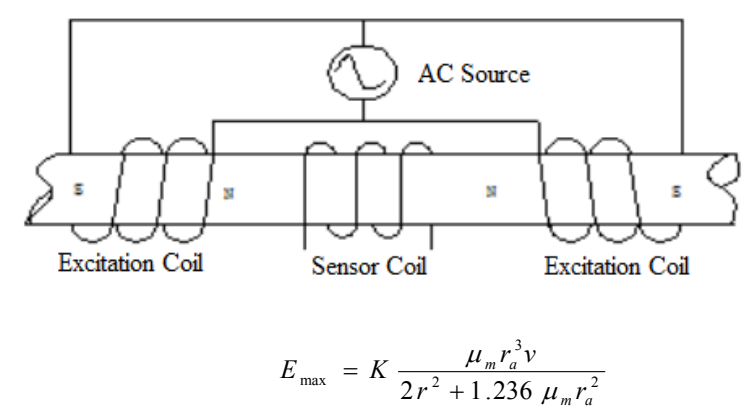

FIGURE I. SENSOR SCHEMATIC

In the formula (1), $r_{a}$ is the radius of the metal particle, $v$ is the flow velocity, $\mu_{m}$ is the relative magnetic permeability and $r$ is the radius of oil duct. The induced voltage of the sensor increases with the increase of the radius of the metal particle [3].

The signal phase of the non-ferromagnetic particles is opposite to the ferromagnetic particles (see Figure 2), which can be used to distinguish the types of wear particles. Therefore, the sensor can monitor the quantity, size and distribution of ferromagnetic and non-ferromagnetic particles.

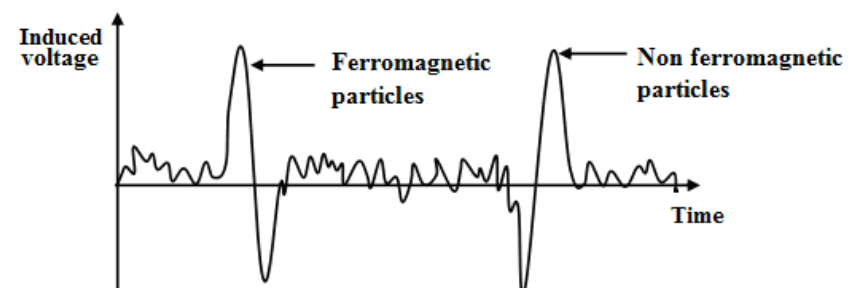

FIGURE II. SENSOR INDUCED VOLTAGE OSCILLOGRAM

\section{COMSOL SimUlation MODELS BUILDING}

\section{A. Two-Dimensional Model}

1) Model building: Due to the symmetry of the sensor structure, so chooses to establish a two-dimensional axisymmetric model. Such Y axis is the symmetry axis. The model establishes a $34 \mathrm{~mm} * 50 \mathrm{~mm}$ rectangle as the air domain, establishes three $5 \mathrm{~mm} * 10 \mathrm{~mm}$ rectangular as three coils, and builds five $0.05 \mathrm{~mm} * 0.025 \mathrm{~mm}$ rectangular as metal particles. The distance between coils is $1 \mathrm{~mm}$. Here the effect of particle velocity is not considered, so the five metal particles in different positions are established, and the results of the wear particles through the sensor are simulated by the frequency 
domain method. The positions of the particles are shown in Figure 3. The results of different models are established in the experiment, and the five models are shown in Figure 4[4].

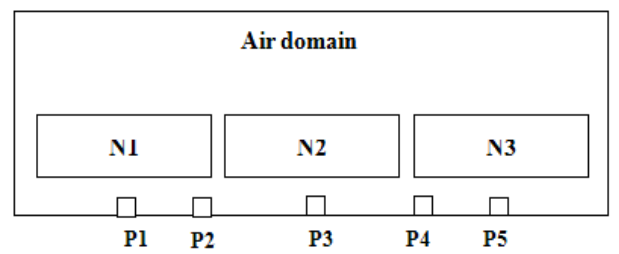

FIGURE III. FIVE LOCATIONS OF PARTICLES

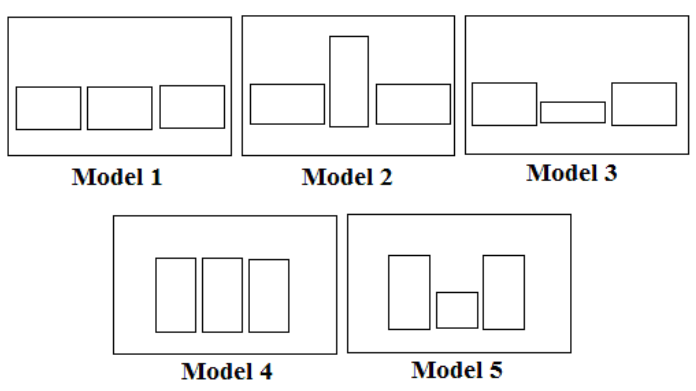

FIGURE IV. 2D FINITE ELEMENT MODELS

2) Material selection: The material of the wire of coils selected copper and set separately in the physical field. The free air field of the coil and the air domain of the model use air material, the relative magnetic permeability of the air material is 1 . In different models, the material of the metal particles selected copper and iron respectively. The relative magnetic permeability of copper material is 0.9999 , and iron material is 4000 .

3) Selection of physical field: The selection of the model physical field is Magnetic Fields (mf). Considering the electromagnetic induced current, the Magnetic Fields (mf) choose Ampere's Law to calculate the magnetic vector potential $\vec{A}$ and electric potential V. Under normal circumstances, COMSOL default the boundary condition of the model is magnetic insulation and electric insulation, and the $\mathrm{Y}$ axis is the axis of symmetry [5]. In order to realize the coupling of electric field and magnetic field, physical fields added two Electrical Circuit (cir).The circuit diagram is shown in Figure 5.

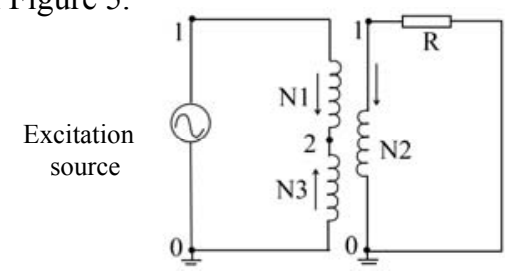

FIGURE V. CIRCUIT DIAGRAM OF 2D MODEL

In Figure 5, the excitation source is a $220 \mathrm{~V} / 2500 \mathrm{~Hz}$ voltage source. In order to make N1 and N3 winding in the opposite direction, the current direction of $\mathrm{N} 1$ and $\mathrm{N} 3$ is in the opposite direction. Resistance $\mathrm{R}$ is $2 \mathrm{k} \Omega$.
The physical field of the coil in the magnetic field is selected as the Multi-Turn Coil, and can modify the number of turns, wire diameter, fill factor and so on. The coupling of electromagnetic fields is required to set the following options: Coil excitation should select circuit (current) option, the coil should select the External I Vs U and the External Device should select the corresponding coil name in the Electrical Circuit field. The parameters of the coils are as follows: N3, $\mathrm{N} 1$ is 500 turns and the wire diameter is $0.2 \mathrm{~mm}$; N2 is 3000 turns and the wire diameter is $0.1 \mathrm{~mm}$. Finally, the physical field is chosen to set the Ampere's Law and modify the relative permeability to define the particle material.

4) Meshing: Considering the air field is set to closer to the real conditions, the coil and the metal particle area choose fine mesh and the air domain use coarse mesh. Then the speed of the model solution would be faster. Mesh as shown in Figure 6.
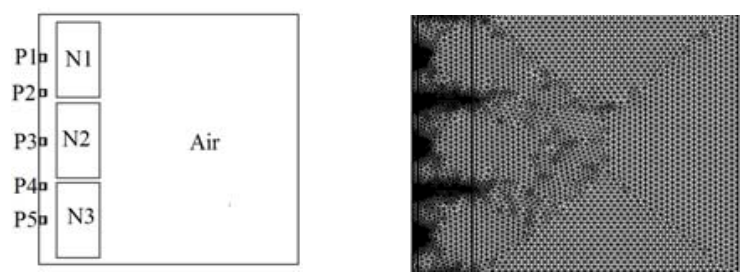

FIGURE VI. MASH MAP

5) Study settings: The study selected Frequency Domain solver. Due to setting of five metal particles at different positions is to simulate the magnetic field change during the metal particle passing through the sensor, so set up five frequency domain solvers, and when we study one of them, we modify the Physics and Variables Selection to shield the other four solvers.

6) Post-treatment of the results: After running Study, in order to get the final results, it is need to carry out the posttreatment of the results. First select the Global Evaluation in the Derived Value option, and then select cir2.IvsU1_v as the expression for the Global Evaluation to calculate the voltage across the coil N2. At last add a 1D Plot Group in the result set, and add five global graphs which expressions are abs (cir2.IvsU1_v). Finally, the global map of the voltage across the coil N2 can be got, which is easy to compare the results.

\section{B. Three-Dimensional Model}

In order to compare the influence on the magnetic field which induced by the different position of the metal particle deviating from the duct center, the 3D model is established.

1) Model building: The air domain is a ball with a diameter of $20 \mathrm{~mm}$ which take $(0,0,0)$ as the center of the sphere. The metal particle is a ball with a diameter of $100 \mu \mathrm{m}$ which take $(0,5,0)$ as the center of the sphere. To build the coil mode, firstly take the $\mathrm{XZ}$ plane as the working plane and draw a circle ring which take $(0,0)$ as the center of the circle in it, and then stretched the ring in the $\mathrm{Y}$ direction. The parameters are as follows: duct diameter is $6 \mathrm{~mm}$, the window 
area of N1 and N3 is $5 \mathrm{~mm} * 5 \mathrm{~mm}$ and N2 is $5 \mathrm{~mm} * 7.5 \mathrm{~mm}$, the distance between coils is $1 \mathrm{~mm}$. The 3D model see Figure 7(a).

Material selection: The material of the coil wire set separately in the physical field. The free air field of the coil and the air domain of the model use air material, the relative magnetic permeability of the air material is 1 . The material of the metal particles selected iron which relative magnetic permeability is 4000 .

2) Selection of physical field: The most important calculation of OMSOL Multiphysics is the magnetic vector potential $\overrightarrow{\mathrm{A}}$, but the most direct result of the simulation is the magnetic induction intensity $\vec{B}$. So the Gauge Fixing for AField is added in the physical field of the 3D model for getting the $\overrightarrow{\mathrm{A}}$ and $\overrightarrow{\mathrm{B}}$.

In order to set the winding direction of the coil, the MultiTurn Coil in the 3D model need to set the Coil Geometry. In this model, the coil type is selected as the circular, then the inner circle is chosen as the winding direction of the coil. The $\mathrm{N} 1$ and N3 are arranged in the opposite direction. There is no effect on the N2 coil winding, so chooses N2 and N1 with same winding direction.

The 3D model also added two Electrical Circuit (cir), see Figure 7(b). In order to reduce the amount of computation, the $10 \Omega$ resistance R1 is added in the Electrical Circuit (cir) 1. Because it has been set up that the coil N1 and N3 is the opposite winding direction in the Coil Geometry, so the two excitation coils $\mathrm{N} 1$ and $\mathrm{N} 3$ can be directly connected in series. Other settings are the same as the 2D axis symmetric model.

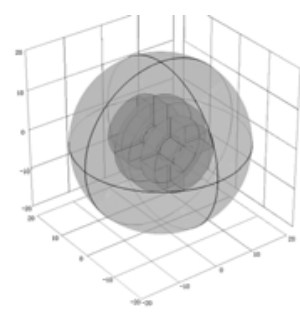

(a) 3D model

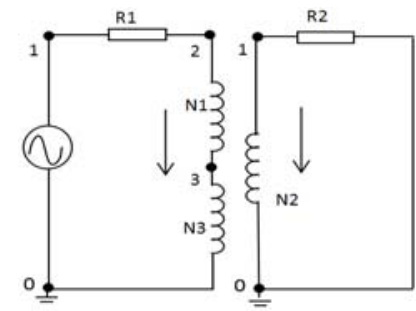

(b) 3D model circuit
FIGURE VII. 3D MODEL ESTABLISHMENT

3) Meshing: Becaus the grid of 3D model has relatively high requirement, and in order to speed up the convergence of the model, the mesh selected Free Tetrahedral. The maximum element size of the coil is $1.3 \mathrm{~mm}$. Due to the small size of the metal particles, the mesh of the particle is needed to be further refined, the maximum element size is $0.05 \mathrm{~mm}$. Similarly, in order to reduce the computation, the air field in the $3 \mathrm{D}$ model is also divided into a coarse grid. Model mesh is shown in Figure 8.

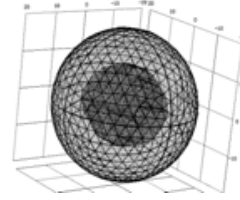

a. Air domain mesh

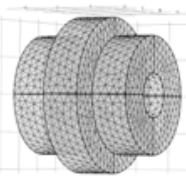

b. Coil mesh

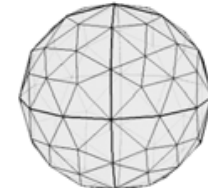

c. Metal particle mesh
FIGURE VIII. 3D MODEL MESH GENERATION
4) Study settings: Compared with the 2D axisymmetric model, 3D model solver settings adds the coil geometry analysis solver, such COMSOL can automatically analyze and calculate the geometric parameters such as the filling coefficient and the center axis of the coil. Other settings are the same as the 2D axisymmetry model. The frequency domain solver is also used.

5) Post-treatment of the results: The 1D plot group of the $3 \mathrm{D}$ model is identical to the $2 \mathrm{D}$ model, but added a $3 \mathrm{D}$ plot group. The 3D plot group is set to draw one $\mathrm{YZ}$ section, and the expression is set to mf.normB to draw the magnetic flux density norm of the model section. In the end, the 1D plot group and the magnetic flux density norm map are obtained.

\section{Simulation RESUlts AND ANALysis}

\section{A. 2D Simulation Results}

1) Influence of particle's position: The model parameters are given below. The diameter of iron particle is $50 \mu \mathrm{m}$; the number of turns of the coil N1, N2 and N3 are 1500, 4500 and 1500 respectively, and the coil wire diameter of them are $0.2 \mathrm{~mm}, 0.1 \mathrm{~mm}$ and $0.2 \mathrm{~mm}$ respectively; the distance between coils is $1 \mathrm{~mm}$; the exciting voltage is $220 \mathrm{~V} / 2500 \mathrm{~Hz}$; the filling coefficient is 0.628 ; the diameter of duct is $6 \mathrm{~mm}$.

Table 1 is the comparison of induced voltages of iron particles in five position of the each model from modellto model 5.

TABLE I. INDUCED VOLTAGES OF DIFFERENT MODEL

\begin{tabular}{rlllll}
\hline Model types & P1 $(\mu \mathrm{V})$ & P2 $(\mu \mathrm{V})$ & P3 $(\mu \mathrm{V})$ & P4 $(\mu \mathrm{V})$ & P5 $(\mu \mathrm{V})$ \\
\hline Mode 1 & 17.123 & 41.448 & $-5.778 \mathrm{e}-3$ & -42.785 & -18.347 \\
Mode 2 & 30.734 & 55.98 & $-4.121 \mathrm{e}-3$ & -56.822 & -31.57 \\
Mode 3 & 13.423 & 39.584 & $-3.959 \mathrm{e}-2$ & -39.92 & -13.587 \\
Mode 4 & 20.288 & 32.488 & $-3.8828 \mathrm{e}-2$ & -32.619 & -20.397 \\
Mode 5 & 21.691 & 36.646 & $-4.8121 \mathrm{e}-3$ & -37.711 & -22.709 \\
\hline
\end{tabular}

The results shown, the bigger induced voltages are in the model 2 when metal particles in position 2(P2) and P4.

2) Influence of excitation frequency: When the particle in P2 of model 2, In addition to the excitation voltage frequency, other model parameters are the same as above A. 1) section. Table 2 is the comparison of induced voltage when the excitation voltage frequency $\mathrm{f} 0$ is as a variable parameter.

TABLE II. INDUCED VOLTAGES OF DIFFERENT EXCITATION FREQUENCY

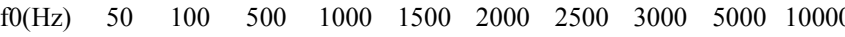

\begin{tabular}{lllllllllll}
$\mathrm{P} 2(\mu \mathrm{V})$ & 0.66 & 2.56 & 30.78 & 46.75 & 51.53 & 53.17 & 53.66 & 53.61 & 51.15 & 40.18 \\
\hline
\end{tabular}

Obviously, the induced voltage is relatively large when the excitation frequency is about $2500 \mathrm{~Hz}$.

3) Influence of turns ratio: In model 2, In addition to the number of turns of the coil N1 and N3(coil N2 keeps 4500 turns), other model parameters are the same as above A. 1) section. Table 3 is the comparison of induced voltages when the excitation coil turn number is as variable parameter.

In Table 3, the larger the turn ratio $(\mathrm{N} 2 / \mathrm{N} 1)$, the bigger the induced voltage. Considering the bearing capacity of sensor 
excitation current, the excitation coil is selected for 500 turns, and the induction coil is selected for 3000 turns finally.

TABLE III. INDUCED VOLTAGE OF DIFFERENT TURNS RATIO

\begin{tabular}{cccccc}
\hline $\mathrm{N} 1, \mathrm{~N} 3$ (turns) & $\mathrm{P} 1(\mu \mathrm{V})$ & $\mathrm{P} 2(\mu \mathrm{V})$ & $\mathrm{P} 3(\mu \mathrm{V})$ & $\mathrm{P} 4(\mu \mathrm{V})$ & $\mathrm{P} 5(\mu \mathrm{V})$ \\
\hline 2000 & 23.226 & 42.291 & -0.311 & -42.925 & -23.85 \\
1500 & 30.745 & 55.98 & -0.412 & -56.823 & -31.57 \\
1000 & 44.93 & 81.82 & -0.6058 & -83.55 & -46.41 \\
500 & 77.24 & 140.6 & -0.11 & -151.7 & -84.3 \\
\hline
\end{tabular}

4) Influence of the distance between coils: In model 2, the coil N2 is 3000 turns, the coil N1 and N3 is 500 turns and the distance between coils is as a variable, other model parameters are the same as above A. 1) section. The comparison of induced voltages is shown in Table 4.

TABLE IV. InduCED Voltage OF DifFerent COIL Distance

\begin{tabular}{cccccc}
\hline Distance $(\mathrm{mm})$ & $\mathrm{P} 1(\mu \mathrm{V})$ & $\mathrm{P} 2(\mu \mathrm{V})$ & $\mathrm{P} 3(\mu \mathrm{V})$ & $\mathrm{P} 4(\mu \mathrm{V})$ & $\mathrm{P} 5(\mu \mathrm{V})$ \\
\hline 1.5 & 46.492 & 85.548 & -0.1765 & -93.708 & -50.792 \\
1 & 50.275 & 91.542 & -0.7423 & -102.37 & -56.875 \\
0.5 & 56.1502 & 98.7615 & -0.143 & -108.87 & -61.76 \\
0.25 & 58.781 & 101.518 & $-3.7381 \mathrm{e}-3$ & -112.345 & -65.042 \\
\hline
\end{tabular}

The smaller the distance is, the bigger the induced voltage.

5) Influence of the duct diameter: In model 2, In addition to three coils turns remain the same and the duck diameter is as a variable, other model parameters are the same as above A. 1) section. The comparison of induced voltages see Table 5.

TABLE V. INDUCED VOLTAGES OF DIFFERENT DUCT DIAMETER

\begin{tabular}{cccccc}
\hline $\begin{array}{c}\text { Diameter } \\
(\mathrm{mm})\end{array}$ & $\mathrm{P} 1(\mathrm{mV})$ & $\mathrm{P} 2(\mathrm{mV})$ & $\mathrm{P} 3(\mathrm{mV})$ & $\mathrm{P} 4(\mathrm{mV})$ & $\mathrm{P} 5(\mathrm{mV})$ \\
\hline 6 & 0.050275 & 0.091542 & $0.7423 \mathrm{e}-6$ & -0.10237 & -0.056875 \\
1 & 0.11732 & 0.269175 & $6.0901 \mathrm{e}-5$ & -0.347541 & -0.151418 \\
0.5 & 0.127526 & 0.301625 & $5.0289 \mathrm{e}-4$ & -0.401397 & -0.169313 \\
0.2 & 0.133907 & 0.323049 & $9.233 \mathrm{e}-5$ & -0.440236 & -0.182097 \\
\hline
\end{tabular}

The smaller the duct diameter is, the bigger the induced voltage.

\section{B. 3D Simulation Results}

Because the 3D model is built to compare the different effects on the magnetic field when the metal particles locate different distances from the radial center of the duct, so in the simulation of $3 \mathrm{D}$ model, the variable parameters are the coordinates of metal particle $(\mathrm{x}, \mathrm{y}, \mathrm{z})$. In this simulation, set up $\mathrm{x}$ and $\mathrm{y}$ as the constant, $\mathrm{x}=0$ and $\mathrm{y}=5$ (the axial direction of the duct as the $\mathrm{Y}$ axis), and the $\mathrm{z}$ value is the distance that the metal particle depart off the radial center of the duct.

The parameters of 3D model are follow: The diameter of iron particle is $100 \mu \mathrm{m}$; the $\mathrm{N} 1$ and $\mathrm{N} 3$ coil turns are 500 and the wire diameter of coils is $0.2 \mathrm{~mm}$; the $\mathrm{N} 2$ coil is 3000 turns and its wire diameter is $0.1 \mathrm{~mm}$; the coil spacing is $1 \mathrm{~mm}$; the exciting voltage is $220 \mathrm{~V} / 2500 \mathrm{~Hz}$; the filling coefficient is 0.628 ; the diameter of duct is $6 \mathrm{~mm}$; the metal particle position is $(0,5, \mathrm{z})$.

The distribution of magnetic flux density in the $\mathrm{YZ}$ section is shown in Figure 9, and the induced voltages of different distance that the metal particle depart off radial center of the duct is shown in Table 6 .

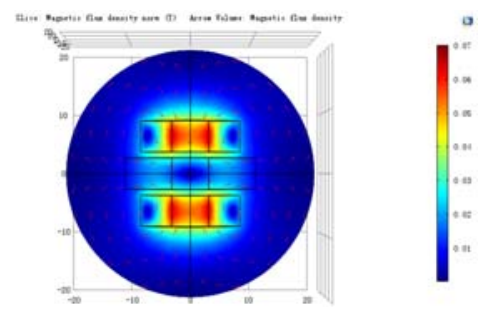

FIGURE IX. YZ SECTION: MAGNETIC FLUX DENSITY NORM(T)

TABLE VI. INDUCTION VOLTAGES OF METAL PARTICLE IN DIFFERENT POSITION

\begin{tabular}{cccccccc}
\hline $\begin{array}{l}\text { Metal Particle } \\
\text { position z }(\mathrm{mm})\end{array}$ & 0 & 0.5 & 1 & 1.5 & 2 & 2.5 & 2.8 \\
\hline Voltage $(\mu \mathrm{V})$ & 26.195 & 25.727 & 27.024 & 49.869 & 57.695 & 46.865 & 49.768 \\
\hline
\end{tabular}

The result is the largest when the particle distances from the radial center of the duct $2 \mathrm{~mm}$.

\section{SUMMARY}

The $2 \mathrm{D}$ and $3 \mathrm{D}$ finite element models are established by using COMSOL Multiphysics software. When the metal particle pass through the sensor coils, the magnetic field of the sensor coils and the characteristics of the voltage of the induction coil are simulated in the different model parameter. The following conclusions are drawn from the simulation experiment:

In the comparison of different models, the Model 2 is better, and the induction voltage is the maximum.

When the frequency of the excitation voltage is about $2500 \mathrm{~Hz}$, the induction voltage is large relatively.

The increasing of turn ratio of induction coil to excitation coil, the decreasing of the coils distance and the duct diameter, all can improve the induction voltage.

In addition, the position of metal particle in the duct (axial and radial), also has an influence on the induced voltage.

The conclusions provide a basis for the research and design of the detection sensor of the metal wear particle in oil fluid.

\section{REFERENCES}

[1] FU Jianting and ZHAN Huiqin, "Detection method of wear particle in oil duct and study on circuit," Master Thesis, University of Electronic Science and Technology of China, 2012, pp. 9-19.

[2] CHEN Shuhan and YAN Hengzhi, "Research in sensing characteristics of reverse-double-excitation solenoid wearing debris sensor," China, J. Instrument Technique and Sensor, 2005, No, 9, pp.6-7.

[3] ZHANG Yijun and YAN Hengzhi, "Research in sensing characteristics of reverse-double-excitation solenoid wearing debris sensor," Master Thesis, Central South University, China, 2004, pp. 23-34.

[4] YAN Hongzhi and ZHANG Yijun, "The design of an on-line monitoring sensor of wear metal particles and the analysis of its characteristic," Chinese Journal of Sensors and Actuators, Vol.15, 2002. pp. 334-338.

[5] WU Deyu and LI Desheng, "Technical study on micro inductive sensor for metal debris static detection," Masteral Dissertation, Beijing University of Technology, China, 2013, pp. 30-34. 